\title{
Tournament Sequences and Meeussen Sequences
}

\author{
Matthew Cook \\ Computational and Neural Systems Program \\ California Institute of Technology \\ Pasadena, CA 91125 \\ cook@paradise.caltech.edu \\ Michael Kleber* \\ Department of Mathematics \\ Massachusetts Institute of Technology \\ Cambridge, MA 02139 \\ kleber@math.mit.edu
}

Submitted: March 22, 2000; Accepted: September 5, 2000

\begin{abstract}
A tournament sequence is an increasing sequence of positive integers $\left(t_{1}, t_{2}, \ldots\right)$ such that $t_{1}=1$ and $t_{i+1} \leq 2 t_{i}$. A Meeussen sequence is an increasing sequence of positive integers $\left(m_{1}, m_{2}, \ldots\right)$ such that $m_{1}=1$, every nonnegative integer is the sum of a subset of the $\left\{m_{i}\right\}$, and each integer $m_{i}-1$ is the sum of a unique such subset.

We show that these two properties are isomorphic. That is, we present a bijection between tournament and Meeussen sequences which respects the natural tree structure on each set. We also present an efficient technique for counting the number of tournament sequences of length $n$, and discuss the asymptotic growth of this number. The counting technique we introduce is suitable for application to other well-behaved counting problems of the same sort where a closed form or generating function cannot be found.
\end{abstract}

MSC: $11 B 99$ (Primary), 05A15, 05A16 (Secondary).

\footnotetext{
*Partially supported by an NSF Mathematical Sciences Postdoctoral Research Fellowship
} 


\section{Introduction}

An infinite tournament sequence $T$ is an infinite sequence of positive integers $T=$ $\left(t_{1}, t_{2}, \ldots\right)$ such that

- $t_{1}=1$ and $t_{i}<t_{i+1} \leq 2 t_{i}$ for $i=1,2, \ldots$

For example, the first infinite tournament sequence in lexicographic order is $t_{i}=i$, and the last is $t_{i}=2^{i-1}$. A finite tournament sequence $T=\left(t_{1}, \ldots, t_{n}\right)$ is a truncated infinite tournament sequence.

An infinite Meeussen sequence $M$ is an infinite sequence of positive integers $M=$ $\left(m_{1}, m_{2}, \ldots\right)$ such that

- $m_{1}=1$ and $m_{i}<m_{i+1}$ for $i=1,2, \ldots$,

- Every nonnegative integer is the sum of a subset of the $\left\{m_{i}\right\}$, and

- Each integer $m_{i}-1$ is the sum of a unique subset of the $\left\{m_{i}\right\}$.

For example, the first infinite Meeussen sequence in lexicographic order is $m_{i}=f_{i+1}$, the $(i+1)$ st Fibonacci number, and the last is $m_{i}=2^{i-1}$. A finite Meeussen sequence $M=\left(m_{1}, \ldots, m_{n}\right)$ is a truncated infinite Meeussen sequence. We will see that this is equivalent to requiring that every integer between 1 and $\sum_{i=1}^{n} m_{i}$ is the sum of a subset of the $\left\{m_{i}\right\}$.

We present a bijection $\{T\} \leftrightarrow\{M\}$ between these two types of sequences. The bijection is defined in Section 2; it preserves the length of the sequence and respects lexicographic ordering. It also acts in a surprising way on sequences with certain recurrence relations, as discussed in Section 3.

Counting finite tournament (or equivalently Meeussen) sequences of length $n$ is straightforward ([9], sequence A008934), but if done in the obvious way takes time exponential in $n$. In Section 4 we present an efficient polynomial-time algorithm for producing the numbers. The technique is suitable for application to other well-behaved counting problems of the same sort where a closed form or generating function cannot be found. We also discuss the asymptotic growth, proving that the $\log _{2}$ of the number of sequences of length $n$ is $\left(\begin{array}{l}n \\ 2\end{array}\right)-\log _{2}(n !)+O\left(\log (n)^{2}\right)$.

Finite tournament sequences were studied in [4] under the name "random knock-out tournaments." A sequence $\left(t_{1}, \ldots, t_{n}\right)$ represented a tournament of $n$ rounds beginning with $1+\sum t_{i}$ players; in the first round $2 t_{n}$ players are paired off randomly and the $t_{n}$ losers are eliminated, leaving a tournament corresponding to $\left(t_{1}, \ldots, t_{n-1}\right)$. The paper concerns the probabilities of certain pairings occurring in such a tournament.

The count which first appeared in [9] was performed by M. Torelli, who found the notion of a tournament sequence useful in his investigation of sequences with certain properties relating to Goldbach's conjecture [10]. Tournament sequences also appear independently in the work of J. Shallit, where they are the possible subword complexities of infinite non-periodic bit strings [11]. 
The observation that the beheaded Fibonacci sequence satisfies the property claimed above was made by Wouter Meeussen [private communication, 1999], and we here name sequences with this property Meeussen sequences in his honor. Part of their definition is similar to that of so-called regular sequences [6], in which each term is a partial sum of preceding terms, which arise in the study of finite probability measures. The unique representability condition is reminiscent of 1-additive sequences (see [7], for example), but the precise form of the condition seems new.

The authors would like to acknowledge W. Meeussen for suggesting the question, and N. J. A. Sloane for his Encyclopedia of Integer Sequences [9], which led us to notice the coincidence. Thanks also to J. Polito for useful conversations, J. Shallit for helpful comments on an earlier draft of this work, and D. Knuth for excellent suggestions about the asymptotics questions.

\section{An Isomorphism on Trees}

In this section we define a map which sends any tournament sequence, finite or infinite, to a Meeussen sequence of the same length. The set of all sequences of either type can naturally be viewed as a rooted tree: the nodes on level $n$ of the tree correspond to the sequences of length $n$, and the parent of the sequence $\left(s_{1}, \ldots, s_{n}\right)$ in the tree is the sequence $\left(s_{1}, \ldots, s_{n-1}\right)$. Our map is an isomorphism on the tree structures of the two types of sequences. Figure 1 shows how the beginnings of these trees look; the node for $\left(s_{1}, \ldots, s_{n}\right)$ has the label $s_{n}$ written on it.

The definition of a tournament sequence $\left(t_{1}, \ldots, t_{n+1}\right)$ says that $t_{n+1}$ can have any value between $t_{n}+1$ and $2 t_{n}$. Therefore the tree of tournament sequences has a convenient local description: the top node is labelled 1 , and any node labelled $k$ has $k$ children, with labels $k+1, k+2, \ldots, 2 k$, respectively. Trees with such local descriptions have been called generating trees, and were introduced in [5]. They have been championed by J. West ([12],[13]), who has used them to study pattern-avoiding permutations, and by Barcucci et. al., who applied them to the enumeration of combinatorial objects [3];

see [2] and references therein for more on the subject. In West's transparent notation, the tree of tournament sequences is

$$
\begin{array}{ll}
\text { Root: } & (1) \\
\text { Rule: } & (k) \rightarrow(k+1)(k+2) \ldots(2 k)
\end{array}
$$

We will prove that the tree of Meeussen sequences is isomorphic to the tree of tournament sequences by showing that it also has the property that if a node has $k$ children, then those children have $k+1, k+2, \ldots, 2 k$ children, respectively. Since this tree structure clearly has no automorphisms, we conclude that there is a unique bijection between the two trees, and therefore a unique bijection between tournament sequences and Meeussen sequences which respects the ideas of extending or truncating a sequence.

To better understand Meeussen sequences, we introduce some notation. 

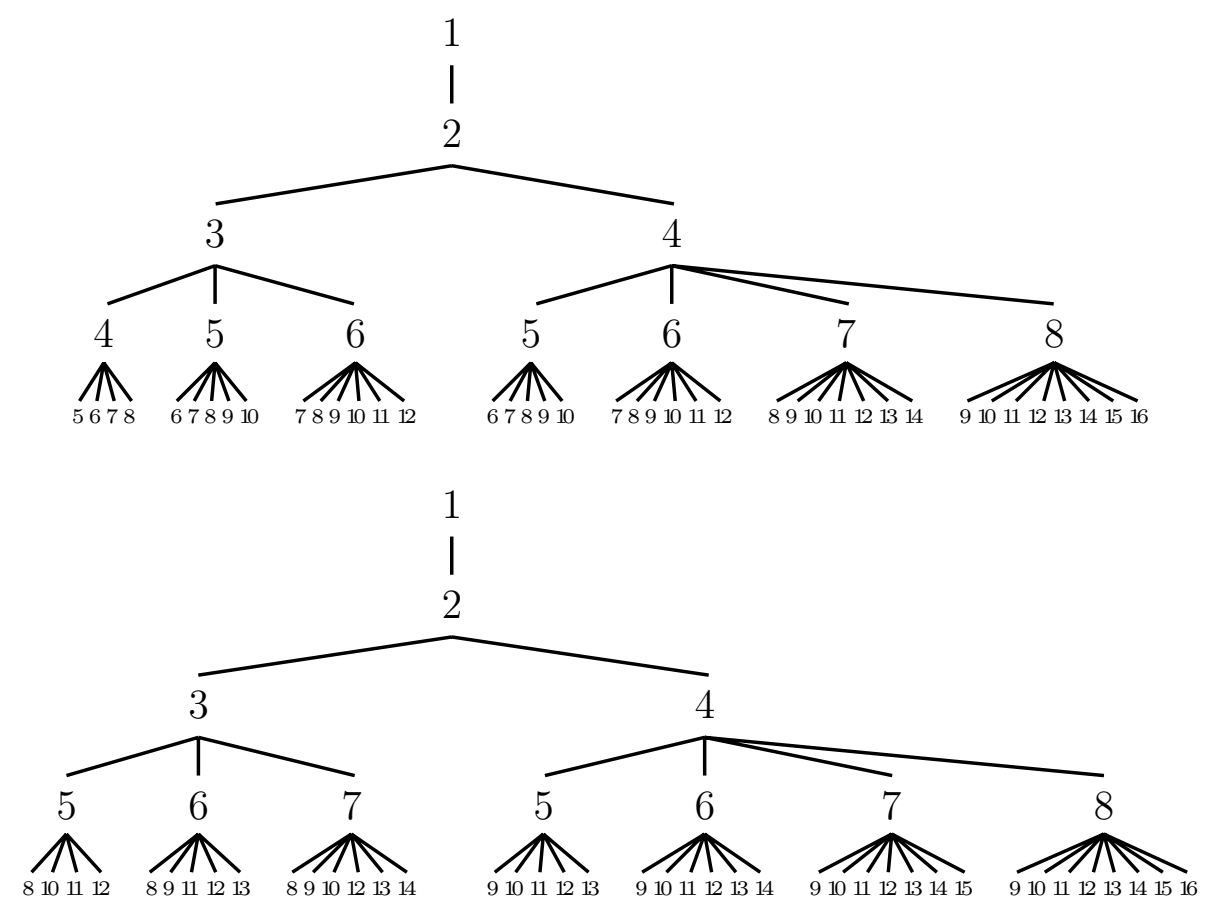

Figure 1: The top five rows of the trees of tournament and Meeussen sequences.

Definition 1 For $A=\left(a_{1}, a_{2}, \ldots\right)$ an integer sequence, finite or infinite, define:

1. $r(A)$ to be the set of integers which are representable as $a_{i_{1}}+\ldots+a_{i_{n}}$ for some $a_{i_{1}}, \ldots, a_{i_{n}}$ in $A, i_{1}<\cdots<i_{n}$, and

2. $\operatorname{ur}(A)$ to be the set of integers so representable in exactly one way.

For example, if $A=(1,2,3)$ is our sequence, then $r(A)=\{0,1,2,3,4,5,6\}$, and $u r(A)=$ $\{0,1,2,4,5,6\}$, where 3 is omitted because it can be represented as 3 and as $1+2$. An infinite increasing integer sequence $M=\left(m_{1}, m_{2}, \ldots\right)$ with $m_{1}=1$ is Meeussen if $r(M)=\mathbb{Z}_{\geq 0}$ and $m_{i}-1 \in u r(M)$ for all $i$.

Given a finite Meeussen sequence $M=\left(m_{1}, \ldots, m_{n}\right)$ which we wish to extend, we must certainly pick $m_{n+1}$ to be $u+1$ for some element $u \in u r(M)$ with $u \geq m_{n}$. We say such choices of $u$ are candidates. For example, with $M=(1,2,3)$, there are three candidates $4,5,6$ for $m_{4}-1$, so $m_{4}$ must be one of 5,6,7.

Our claim that the two trees are isomorphic then reduces to the following:

Proposition 2 Suppose $M=\left(m_{1}, \ldots, m_{n}\right)$ is a finite Meeussen sequence, and there are $k$ candidates for $m_{n+1}-1$, which we designate $u_{1}<u_{2}<\cdots<u_{k}$. Then for each $j$, $1 \leq j \leq k$, the extended sequence with $m_{n+1}=u_{j}+1$ is also Meeussen, and has $k+j$ candidates for $m_{n+2}-1$. 
Proof: Let $S$ denote the sum $\sum_{i=1}^{n} m_{i}$ of the sequence; note that $S$ is also the largest candidate, $u_{k}$. Let $M^{\prime}=\left(m_{1}, \ldots, m_{n+1}\right)$ be the extended sequence we get by choosing $m_{n+1}=u_{j}+1$, and $S^{\prime}=S+m_{n+1}$ be its sum.

First, we can see by induction that $r\left(M^{\prime}\right)$ is the entire interval of integers $\left[0, S^{\prime}\right]$. Each representable sum in $r\left(M^{\prime}\right)$ is an element of $r(M)$ or is $m_{n+1}$ added to such an element. Assume inductively that each of these types forms a single interval, $[0, S]$ and $\left[m_{n+1}, S^{\prime}\right]$, respectively. There is no gap between the two intervals, since $m_{n+1}-1=u_{j} \in r(M)$, and the induction holds.

Note that these two intervals have some overlap $\left[m_{n+1}, S\right]$, possibly empty if we chose $m_{n+1}=u_{k}+1=S+1$. Anything in the overlap can be represented in at least two ways, one with $m_{n+1}$ and one without. Thus the candidates for $M^{\prime}$, the elements of $u r\left(M^{\prime}\right)$ larger than $m_{n+1}$, are in fact all larger than $S$.

Next we observe that both $r\left(M^{\prime}\right)$ and $u r\left(M^{\prime}\right)$ are invariant under the involution $t \mapsto S^{\prime}-t$, corresponding to taking the complement of a subset. The overlap $\left[m_{n+1}, S\right]$ is similarly invariant and right in the middle, and for each candidate $v$ of $M^{\prime}$, there will be a corresponding member $S^{\prime}-v$ of $u r\left(M^{\prime}\right)$ smaller than $m_{n+1}$.

Similarly, $\operatorname{ur}(M)$ has the same property: aside from $u_{1}, \ldots, u_{k}$ it contains exactly $k$ more uniquely representable numbers, all of the form $S-u_{i}$. We now know that the elements of $u r(M)$ are, in order, $S-u_{k}, \ldots, S-u_{1}, u_{1}, \ldots, u_{k}$.

We have also concluded that the candidates for $M^{\prime}$ are exactly the numbers of the form $u+m_{n+1}$ such that $u \in u r(M)$ and the total is strictly larger than $S$. Therefore when we chose $m_{n+1}=u_{j}+1$, the candidates for $M^{\prime}$ are exactly the $k$ numbers $u_{i}+u_{j}+1$ for $i=1, \ldots, k$ and the $j$ numbers $\left(S-u_{i}\right)+u_{j}+1$ for $i=1, \ldots, j$. Thus there are $k+j$ candidates, as desired.

Note that we incidentally showed that half of our definition of Meeussen sequences is unnecessary. If we always choose the term $m_{n+1}$ to be one more than a representable sum from $r(M)$, we argued above that at each finite stage, $r(M)$ is the entire interval $[0, S]$. Thus an infinite sequence $M$ generated this way will automatically have $r(M)=\mathbb{Z}_{\geq 0}$, a condition imposed in the original definition.

Corollary 3 There is a unique isomorphism $\phi:\{T\} \rightarrow\{M\}$ from the set of tournament sequences to the set of Meeussen sequences which preserves the rooted tree structure. Moreover, $\phi$ respects the lexicographic ordering on sequences of each type.

Proof: By Proposition 2, the tree structure of Meeussen sequences is precisely that of tournament sequences: both trees start with a node with one child, and if a node has $k$ children, then those children have $k+1, k+2, \ldots, 2 k$ children, respectively. Since the children of a node are all distinguishable from one another, by virtue of their distinct numbers of children, there is a unique bijection between the two trees.

The nodes in the trees are indexed by finite sequences of each type, so $\phi$ is immediately defined for finite sequences. In both trees, the distance from the root determines the length of the sequence, so $\phi$ preserves it. Infinite sequences can be thought of as 
infinite paths in the tree heading away from the root, and therefore the tree bijection lets us define $\phi$ in this case as well.

It is evident that $\phi$ respects the lexicographic ordering since, in the proof of Proposition 2 , we showed that when extending a sequence $M$, the larger candidates correspond to the nodes with more children, just as in tournament sequences.

The proof of Proposition 2 also gives us a way to calculate $\phi(T)$ for any $T=$ $\left(t_{1}, \ldots, t_{n}\right)$ without constructing the full set $u r(M)$, a task that could require exponential time. We make repeated use of the fact that the candidates for extending $M=\left(m_{1}, \ldots, m_{n}\right)$ are easily expressed in terms of the candidates for $\left(m_{1}, \ldots, m_{n-1}\right)$. Letting $u(n, k)$ be the $k$ th smallest candidate for extending $\left(m_{1}, \ldots, m_{n}\right)$, we get the following recurrence:

$$
\begin{aligned}
m_{n} & =u\left(n-1, t_{n}-t_{n-1}\right)+1, \text { and } \\
u(n, k)= & \begin{cases}u\left(n-1, k-\left(t_{n}-t_{n-1}\right)\right), & \text { if } k>t_{n}-t_{n-1} \\
S_{n}-u\left(n-1, t_{n}-t_{n-1}+1-k\right), & \text { if } k \leq t_{n}-t_{n-1} \\
\text { where } S_{n}=m_{1}+\cdots+m_{n} .\end{cases}
\end{aligned}
$$

Beginning with $m_{1}=1$ and $u(1,1)=1$, we can quickly calculate each successive term of $\phi(T)$ in linear time.

\section{Properties of the Bijection}

In the introduction, we stated without proof that the beheaded Fibonacci sequence $(1,2,3,5,8,13, \ldots)$ is a Meeussen sequence, and moreover that it is the smallest one in lexicographic order, the image of $(1,2,3,4,5,6, \ldots)$ under the map $\phi$ defined above. This is a special case of a more general surprising property of $\phi$, which we prove in this section.

Since $(1,2,3,5,8,13, \ldots)$ is, coincidentally, again a tournament sequence, we can apply $\phi$ to it as well. This leads to the following computational observation:

$$
(1,2,3,5,8,13,21, \ldots) \stackrel{\phi}{\longmapsto}(1,2,3,6,11,20,37, \ldots) \stackrel{\phi}{\longmapsto}(1,2,3,7,13,25,48, \ldots)
$$

The middle sequence is the " 3 -bonacci" sequence beginning $(1,2,3)$ and the last is the "4-bonacci" sequence beginning $(1,2,3,7)$, where we say a sequence is $k$-bonacci if each term (after the first $k$ ) is the sum of the previous $k$ terms. Further experimentation reveals that, for example,

$$
(1,2,4,7,12,20,33,54,88,143, \ldots) \stackrel{\phi}{\mapsto}(1,2,4,7,13,24,44,81,149,274, \ldots) .
$$

The first sequence begins $(1,2)$ and thereafter each term is one plus the sum of the previous two, while the second sequence is the 3 -bonacci sequence beginning $(1,2,4)$, with no additive constant in the recurrence.

Inspired by examples of this type, we make the following observation. 
THE ELECTRONIC JOURNAL OF COMBINATORICS 7 (2000), \#R44

Proposition 4 Suppose $\left(t_{1}, \ldots, t_{n+1}\right)$ is a finite tournament sequence, and suppose that for some integers $k, c$, it happens that both

$$
\begin{aligned}
& t_{n+1}=t_{n}+t_{n-1}+\cdots+t_{n-k+1}+c \quad \text { and } \\
& t_{n}=t_{n-1}+t_{n-2}+\cdots+t_{n-k}+c .
\end{aligned}
$$

Then $\left(t_{1}, \ldots, t_{n+1}\right) \stackrel{\phi}{\longmapsto}\left(m_{1}, \ldots, m_{n+1}\right)$, where

$$
m_{n+1}=m_{n}+m_{n-1}+\cdots+m_{n-k+1}+m_{n-k} .
$$

While the statement of the proposition is designed to mimic the examples above, the hypothesis simplifies to $t_{n+1}=2 t_{n}-t_{n-k}$. Since our map $\phi$ respects extending or truncating a sequence, we are really just assuming that $t_{n+1}=2 t_{n}-t_{n-k}$ for some single pair $n, k$; the effect is purely local.

To prove the proposition, it would help to have a more concrete relationship between terms of the tournament and Meeussen sequences associated to one another by our bijection.

Lemma 5 Let $T=\left(t_{1}, t_{2}, \ldots\right)$ be a (finite or infinite) tournament sequence with associated Meeussen sequence $\phi(T)=M=\left(m_{1}, m_{2}, \ldots\right)$. Write ur $(M)$, the uniquely representable sums of $M$, as $\left\{u_{1}<u_{2}<u_{3}<\cdots\right\}$. Then:

1. The $t_{i}$ 'th uniquely representable sum $u_{t_{i}}$ is $m_{i}-1$, and

2. The next uniquely representable sum $u_{t_{i}+1}$ is $m_{1}+m_{2}+\cdots+m_{i-1}+1$.

\section{Proof:}

1. When $i=1$, we check that $t_{1}=1, u_{1}=0$, and $m_{1}=1$ directly. Now assume by induction that the map $k \mapsto 1+u_{k}$ sends $t_{i}$ to $m_{i}$ for some $i$. Then it sends $t_{i}+1, t_{i}+2, \ldots$ to the first, second, .. number in $u r(M)$ greater than $m_{i}$, which we showed was the desired value during the proof of Proposition 2.

2. Consider the truncated sequence $M_{i}=\left(m_{1}, \ldots, m_{i}\right)$. The map $t \mapsto\left(m_{1}+\cdots+\right.$ $\left.m_{i}\right)-t$, as we saw in the proof of Proposition 2, is an involution on $u r\left(M_{i}\right)$, and takes $m_{i}-1$ to $m_{1}+\cdots+m_{i-1}+1$. Certainly nothing in between is uniquely representable; this is the "overlap" interval of numbers which can be represented either with or without using $m_{i}$. This in turn means that $m_{i+1}$ is strictly larger than $m_{1}+\cdots+m_{i-1}+1$, which is therefore in $u r(M)$ since it is in $u r\left(M_{i}\right)$.

Proof of Proposition 4: Consider $M=\left(m_{1}, \ldots, m_{n}\right)$ with sum $S=m_{1}+\cdots+m_{n}$ and $\operatorname{ur}(M)=\left\{u_{1}<u_{2}<u_{3}<\cdots\right\}$. By the first part of Lemma 5, we know that $m_{n}=1+u_{t_{n}}$. Therefore $u r(M)$ contains exactly $2 t_{n}$ numbers: $u_{1}<\cdots<u_{t_{n}}$, which are less than $m_{n}$, and another $t_{n}$ which are their images under the involution $t \mapsto S-t$. In particular, $u_{2 t_{n}-i}=S-u_{i+1}$. 
THE ELECTRONIC JOURNAL OF COMBINATORICS 7 (2000), \#R44

Now consider what happens when we pick $t_{n+1}=2 t_{n}-t_{n-k}$, as supposed by Proposition 4 , and extend $M$ accordingly:

$$
\begin{aligned}
m_{n+1} & =1+u_{t_{n+1}} \text { by Lemma } 5 \\
& =1+u_{2 t_{n}-t_{n-k}} \\
& =1+S-u_{t_{n-k}+1} \\
& =1+S-\left(m_{1}+\cdots+m_{n-k-1}+1\right) \text { by Lemma } 5 \text { again, } \\
& =m_{n}+m_{n-1}+\cdots+m_{n-k}
\end{aligned}
$$

The new term of $M$ is the sum of the previous $k$ terms, as claimed.

\section{The Growth of the Tree}

We would like to know the number of tournament or Meeussen sequences of length $n$, which we will designate $s(n)$. Equivalently, we want to know the number of nodes on the $n$th level of the tree shown in Figure 1 (p. 4), where we can count that $s(n)=1,1,2,7,41$ for $n$ up to 5 . Counting the nodes directly takes time exponential in $n$, and while we cannot present a solution in closed form, we can offer an efficient polynomial-time algorithm. We would also like to know the asymptotic behavior of $s(n)$ as $n$ gets large. The asymptotics reveal that $s(n)$ grows so quickly that its generating function cannot be algebraic.

\section{Exact Counting}

Throughout this section, we consider the nodes to be labelled as in the tree of tournament sequences: a node with label $(k)$ has $k$ children, with labels $(k+1),(k+2), \ldots,(2 k)$, respectively. Based on this definition, we note that the function $c(n, k)$ counting the number of nodes with label $(k)$ in row $n$ of the tree satisfies:

\section{Recurrence 1}

$$
\begin{aligned}
& c(1, k)=\delta_{k, 1} \\
& c(n, k)=\sum_{j=\left\lceil\frac{k}{2}\right\rceil}^{k-1} c(n-1, j) \quad \text { for } n>1 .
\end{aligned}
$$

We could then find the number of nodes on row $n$ by summing $c(n, k)$ for all $k$ up to $2^{n-1}$. The work involved grows exponentially in $n$, though, so for large $n$ this is impractical. We could define a generating function in two independent variables

$$
g(x, y)=\sum_{n, k} x^{n} y^{k} c(n, k)=x y+x^{2} y^{2}+x^{3}\left(y^{3}+y^{4}\right)+\cdots
$$


which, based on Recurrence 1, must satisfy

$$
g(x, y)=x y+\frac{x y}{1-y} g(x, y)-\frac{x y}{1-y} g\left(x, y^{2}\right)
$$

Rewriting this as $g(x, y)=\frac{x y(y-1)}{x y+y-1}+\frac{x y}{x y+y-1} g\left(x, y^{2}\right)$, we can solve formally by iterated substitution to get

$$
g(x, y)=\sum_{n=0}^{\infty}\left(y^{2^{n}}-1\right) \prod_{k=0}^{n} \frac{x y^{2^{k}}}{x y^{2^{k}}+y^{2^{k}}-1}
$$

This seems to offer dim prospects for a nice form for $s(n)$, the coefficient of $x^{n}$ at $y=1$.

Alternatively, one could hope to work with the function $d(n, k)$ which counts the number of $n$ th-generation descendents of a node labelled $(k)$. We can count these descendents by summing the number of $(n-1)$-generation descendents of the node's $k$ children:

\section{Recurrence 2}

$$
\begin{aligned}
& d(1, k)=k \quad \text { for all } k \geq 1, \\
& d(n, 0)=0 \quad \text { for all } n \geq 1, \text { and otherwise, } \\
& d(n, k)=\sum_{j=k+1}^{2 k} d(n-1, j) .
\end{aligned}
$$

The number of nodes on row $n$ of our tree is then $s(n)=d(n-1,1)$. Torelli points out that this recurrence can be expressed in closed form, by replacing the last line with

$$
d(n, k)=d(n, k-1)-d(n-1, k)+d(n-1,2 k-1)+d(n-1,2 k)
$$

This alternate version embodies the notion that the tree below a node labelled $(k)$ looks just like the tree below a $(k-1)$, but modified by pruning the branch beginning with the child $(k)$ and grafting on branches beginning with $(2 k-1)$ and $(2 k)$ instead. However, as $n$ increases, either version still involves calculating an exponentially growing set of values.

We offer instead the following technique for calculating the growth of the tree in polynomial time. Consider the family of functions $p_{n}$ for $n=1,2,3, \ldots$ such that $p_{n}(k)$ is the number of $n$ th-generation descendents of a node labelled $(k)$, what we called $d(n, k)$ above. Then in the spirit of Recurrence 2 , we can get a recurrence relation for the functions $p_{n}$ themselves:

\section{Recurrence 3}

$$
\begin{aligned}
& p_{1}(k)=k \\
& p_{n}(k)=\sum_{j=k+1}^{2 k} p_{n-1}(j)
\end{aligned}
$$


Purists would start the recurrence with $p_{0}(k)=1$ instead.

The key observation is that each $p_{n}$ is in fact a degree $n$ polynomial in $k$, which we obtain by symbolic summation of a range of values of $p_{n-1}$. Recall that the sum $\sum_{j=1}^{k} j^{n}$ is a polynomial in $k$ of degree $n+1$. Our recurrence states that $p_{n}(k)$ is the sum of the first $2 k$ values of $p_{n-1}$ minus the sum of the first $k$ values. Since $p_{n-1}$ is a polynomial in $k$ by induction, so is $p_{n}$. The next few polynomials after $p_{1}=k$ are

$$
p_{2}=\frac{3 k^{2}+k}{2}, \quad p_{3}=\frac{7 k^{3}+6 k^{2}+k}{2}, \quad p_{4}=\frac{105 k^{4}+154 k^{3}+63 k^{2}+6 k}{8}, \ldots
$$

Modern computer algebra packages can generally carry out this type of symbolic summation quickly, so we can use this polynomial recurrence directly to find $p_{n}$, and evaluate $p_{n-1}(1)$ to find the number of nodes on level $n$. For example, in Maple ${ }^{\mathrm{TM}}$ :

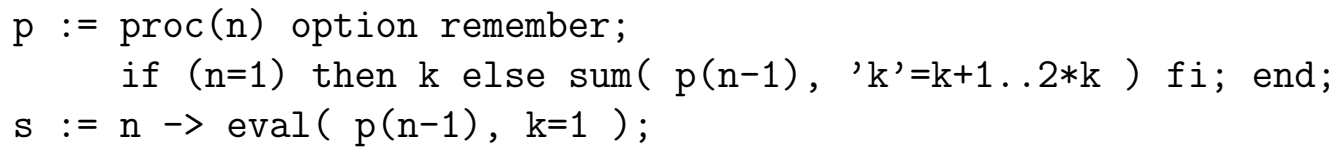

Then $\mathrm{p}(\mathrm{n})$ returns the polynomial $p_{n}$ in the variable $\mathrm{k}$, and $\mathrm{s}(\mathrm{n})$ evaluates $p_{n-1}$ at $k=1$, giving us the number of nodes on level $n$ of the tree.

Now that we know that $p_{n}$ is an $n$ th-degree polynomial, we need not calculate the polynomial explicitly just to find some of its values. For example, $p_{n}$ is determined by its values at the $n+1$ points $k=0,1, \ldots, n$, which we can find (using Recurrence 2) once we know $p_{n-1}$ at $k=0,1, \ldots, 2 n$. We could then fit an interpolating polynomial to those points to find other desired values of $p_{n}$. In this case, another trick presents itself; we can use the linear dependence among $n+2$ equally-spaced values of a polynomial $p$ of degree $n$ :

$$
\forall a, b: \sum_{i=0}^{n+1}(-1)^{i}\left(\begin{array}{l}
n \\
i
\end{array}\right) p(a+b i)=0 .
$$

Combining all of these tricks, we can efficiently calculate the number of nodes on row $n$ of our tree as follows:

\section{Recurrence 4}

$$
\begin{aligned}
& d(0, k)=1 \quad \text { for all } k, \\
& d(n, 0)=0 \quad \text { for all } n>0, \\
& d(n, k)=d(n, k-1)-d(n-1, k)+d(n-1,2 k-1)+d(n-1,2 k) \\
& \quad \text { for } k \leq n, \text { and }
\end{aligned}
$$


As before, $s(n)=d(n-1,1)$ gives the number of nodes on level $n$ of our tree.

Now that we have a refined computational technique, we would like to evaluate its efficiency and justify our statement that it can calculate $s(n)$ in time polynomial in $n$. The difficulty here is that we cannot in good conscience simply count the number of arithmetic operations involved in finding $d(n-1,1)$ using Recurrence 4 , because the values we encounter grow quickly as $n$ increases. Observe, for example, that $s(n)$ certainly grows faster than $c^{n}$ for any fixed constant $c$, because on row $n$ of the tree, every node has at least $n$ (and at most $2^{n-1}$ ) children.

In this situation, an analysis of algorithmic complexity must take into account the magnitude of the numbers involved in arithmetic operations. Bach and Shallit [1] argue for what they call the naive bit complexity measure, in which we can calculate $a+b$ and $a b$ in time $O(\log a+\log b)$ and $O(\log a \log b)$, respectively. These time estimates reflect the speed of the naive, grade-school algorithms for adding and multiplying two numbers with $\log a$ and $\log b$ digits; the authors argue that these estimates are both asymptotically realistic and pragmatic for predicting real-world behavior of computations.

Theorem 6 The naive bit complexity of calculating $s(n)$ is $O\left(n^{6}\right)$.

Note that this is not what one might generally call a polynomial-time computation, since it is polynomial in $n$, not in $\log n$, the length of the input. As already mentioned, $s(n)$ grows so quickly that it could not even be written down in time polynomial in $\log n$.

To calculate $s(n)$, we will compute all values $d(m, k)$ for $0 \leq m \leq n-1$ and $0 \leq k \leq$ $2 m+2$, in lexicographic order, using Recurrence 4 . We first need some bound on the size of the numbers we encounter.

Lemma $7 \quad d(n, k) \leq 2^{n(n-1) / 2} k^{n}$

Proof: The bound is based on Recurrence $2, d(n, k)=\sum_{j=k+1}^{2 k} d(n-1, j)$. For a fixed $n \geq 1$, we know $d(n, k)$ is an increasing function on positive integers $k$. Therefore the largest of the $k$ terms in the sum is $d(n-1,2 k)$, and we have $d(n, k) \leq k d(n-1,2 k)$. Repeating, we get:

$$
\begin{aligned}
d(n, k) \leq & k d(n-1,2 k) \leq(k)(2 k) d(n-2,4 k) \leq \cdots \\
\leq & (k)(2 k) \cdots\left(2^{n-1} k\right) d\left(0,2^{n} k\right) \\
& =2^{n(n-1) / 2} k^{n}
\end{aligned}
$$

In the next section we will discuss the asymptotic growth of $s(n)$, which we have just bounded by $2^{\left(\begin{array}{c}n-1 \\ 2\end{array}\right)}$.

Proof of Theorem 6: We will perform the calculation using Recurrence 4, storing partial results so we never calculate any value of $d$ twice.

Fix some $n$, and suppose we know $d(n-1, k)$ for all $0 \leq k \leq 2 n$. Calculating $d(n, k)$ for all $0 \leq k \leq 2 n+2$ involves two phases. 
1. For each $k$ with $0 \leq k \leq n$, we compute the sum of four numbers. By Lemma 7 , the summands are of length $O\left(n^{2}+n \log k\right)$, which is $O\left(n^{2}\right)$ since $k$ is small. Thus each value of $k$ takes time $O\left(n^{2}\right)$, and the whole phase takes time $O\left(n^{3}\right)$.

2. For $n+1 \leq k \leq 2 n+2$, we compute $\sum_{j=1}^{n+1}(-1)^{(j-1)}\left(\begin{array}{c}n+1 \\ j\end{array}\right) d(n, k-j)$. As above, $d(n, k-j)$ has length $O\left(n^{2}\right)$. The binomial coefficient $\left(\begin{array}{c}n+1 \\ j\end{array}\right)$ has length $O(n)$, since numbers in the $n$th row of Pascal's Triangle are bounded by $2^{n}$; the cost of computing it is negligible because we are using all of the top $n$ rows of Pascal's Triangle, which we can compute by additive recurrence.

Then we form each product in time $O\left(n^{3}\right)$ and take their sum in time $O\left(n^{4}\right)$ for each $k$. Thus the whole phase takes time $O\left(n^{5}\right)$.

Thus passing from $n-1$ to $n$ takes time $O\left(n^{5}\right)$, and we can calculate $s(n)$ from scratch in time $O\left(n^{6}\right)$.

In practice, Recurrence 4 is easy to implement and seems to perform much better than the above analysis suggests for values of $n$ we are interested in. In Maple ${ }^{\mathrm{TM}}$ :

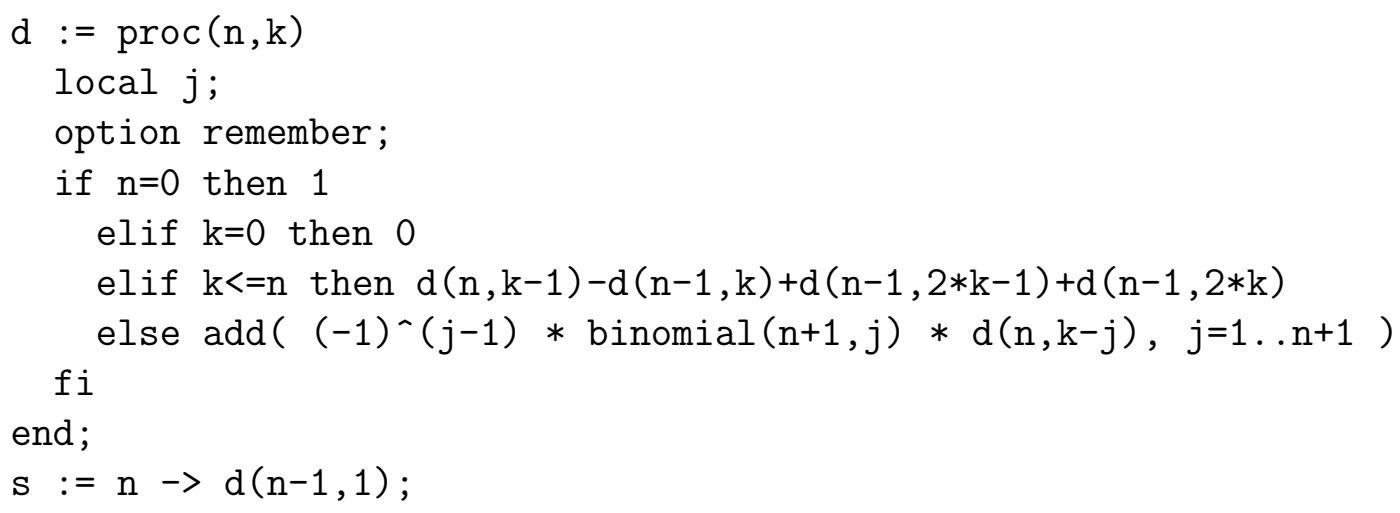

This code seems empirically to calculate $s(n)$ after having done the work for $s(n-1)$ in time $O\left(n^{2}\right)$ even when $n$ is around 190, when $s(n)$ has over 5000 decimal digits. This is the behavior one would expect if multiplication had a constant unit cost and addition were free.

On a modest desktop Pentium II, this computes up to $s(30)$ in under a second, $s(85)$ in under a minute, and s(190) in about an hour; a little extra work to avoid computing the binomial coefficients multiple times speeds it up even more. We record $s(n)$ for $1 \leq n \leq 22$ here. The sequence also appears as entry A008934 in Sloane's On-Line Encyclopedia of Integer Sequences [9].

1, 1, 2, 7, 41, 397, 6377, 171886, 7892642, 627340987, 87635138366, 21808110976027, 9780286524758582, 7981750158298108606, 11950197013167283686587,33046443615914736611839942 , 169758733825407174485685959261,1627880269212042994531083889564192 , 29264239787495935863325877024506142042 , 


$$
\begin{aligned}
& 989901541366810465070950556260422637919176, \\
& 63214893835996484808167529681187283166038800097, \\
& 7643667309922877343580868981767361594845888953165967, \ldots
\end{aligned}
$$

\section{Asymptotic Behavior}

Now we turn to the asymptotic growth of $s(n)$. We know from Lemma 7 that $\left(\begin{array}{c}n-1 \\ 2\end{array}\right)$ is an upper bound for $\lg s(n)$, where $\lg$ denotes $\log _{2}$. We will first prove that a lower bound for $s(n)$ is $\alpha 2^{\left(\begin{array}{c}n \\ 2\end{array}\right)} /(n-1)$ ! for a certain constant $\alpha$, and then we will show that $\lg s(n)$ is asymptotic to $\left(\begin{array}{l}n \\ 2\end{array}\right)-\lg n !+O(\log n)^{2}$.

We are grateful to Donald Knuth for suggesting the method used here. The technique rests on the following observation:

Theorem 8 (Knuth) Let $T$ be a rooted tree in which we want to know the number of vertices on the $n$th level. Select $n-1$ vertices $v_{1}, \ldots, v_{n-1}$ in $T$ by choosing $v_{1}$ to be the root and picking $v_{i+1}$ uniformly at random from among the children of $v_{i}$, of which there are $\operatorname{deg}\left(v_{i}\right)$. Then the expected value

$$
E\left(\operatorname{deg}\left(v_{1}\right) \operatorname{deg}\left(v_{2}\right) \cdots \operatorname{deg}\left(v_{n-1}\right)\right)
$$

is exactly the number of vertices on the nth level of $T$.

Proof: See [8] for a discussion of this technique in greater generality. In this case, a proof by induction is straightforward: if this technique works for counting the $n$th level of each of $k$ trees $T_{1}, \ldots, T_{k}$, then it clearly also works for counting level $n+1$ of the tree $T$ whose root has $k$ children, the roots of $T_{1}, \ldots, T_{k}$.

We will apply Theorem 8 to the tree of tournament sequences, in which, conveniently, each vertex is already labelled with its degree. This means we can calculate $s(n)$ by finding the expected value of the product $t_{1} t_{2} \cdots t_{n-1}$, where $\left(t_{1}, t_{2}, \ldots, t_{n-1}\right)$ is a tournament sequence selected at random by setting $t_{1}=1$ and picking $t_{i+1}$ uniformly at random from among $t_{i}+1, t_{i}+2, \ldots, 2 t_{i}$. For the remainder of this section, whenever we talk about a distribution for $t_{i}$, it is implicitly with respect to this way of picking a tournament sequence.

Lemma 9 Let $s(n)$ be the number of tournament sequences of length $n$. Then

$$
s(n) \geq \alpha \frac{2^{\left(\begin{array}{c}
n \\
2
\end{array}\right)}}{(n-1) !}
$$

where $\alpha=\left(1-\frac{1}{2}\right)\left(1-\frac{1}{4}\right)\left(1-\frac{1}{8}\right) \cdots \approx .28878837 \ldots$ 
Proof: Observe that there is a natural continuous analogue to the expected value $E\left(t_{1} t_{2} \cdots t_{n-1}\right)=s(n)$. Consider instead the expected value $E\left(r_{1} r_{2} \cdots r_{n-1}\right)$, where $r_{1}=1$ and $r_{i+1}$ is a real number chosen uniformly at random from the interval $\left(r_{i}, 2 r_{i}\right]$. Equivalently, we are taking random variables $u_{i}(1 \leq i \leq n-2)$ each with a uniform distribution over $(1,2]$, and setting $r_{i+1}=u_{i} r_{i}$.

The expected value in the continuous case will give an underestimate of the expected value for the discrete version, in which the $u_{i}$ are distributed the same way but we set $t_{i+1}=\left\lceil u_{i} t_{i}\right\rceil$. The independence of the various $u_{i}$ make the expected value easy to calculate:

$$
\begin{aligned}
E\left(r_{1} r_{2} r_{3} \cdots r_{n-1}\right) & =E\left((1)\left(u_{1}\right)\left(u_{1} u_{2}\right) \cdots\left(u_{1} u_{2} \cdots u_{n-2}\right)\right) \\
& =E\left(u_{1}^{n-2} u_{2}^{n-3} \cdots u_{n-2}^{1}\right) \\
& =\frac{2^{n-1}-1}{n-1} \frac{2^{n-2}-1}{n-2} \cdots \frac{2^{2}-1}{2} \\
& \geq \alpha \frac{2^{\left(\begin{array}{c}
n \\
2
\end{array}\right)}}{(n-1) !}
\end{aligned}
$$

Now we show that this lower bound is quite good, by finding the rate of growth of the error. We use lg to denote $\log _{2}$.

Theorem $10 \quad \lg s(n)=\left(\begin{array}{l}n \\ 2\end{array}\right)-\lg n !+O(\log n)^{2}$

Proof: In the proof of Lemma 9, we calculated $E\left(u_{k}^{n-k-1}\right)$, where $u_{k}=r_{k+1} / r_{k}$ was uniformly distributed over $(1,2]$. In the original problem, $t_{k}$ and $t_{k+1}$ are integers, so the ratio $t_{k+1} / t_{k}$ instead takes on a discrete set of values, each with equal probability:

$$
E\left(\left(\frac{t_{k+1}}{t_{k}}\right)^{p}\right)=\frac{\left(\frac{t+1}{t}\right)^{p}+\left(\frac{t+2}{t}\right)^{p}+\cdots+\left(\frac{t+t}{t}\right)^{p}}{t}
$$

where $t=t_{k}$ throughout. We can rewrite this and take advantage of the ability to sum consecutive $p$ th powers:

$$
\begin{aligned}
E\left(\left(\frac{t_{k+1}}{t_{k}}\right)^{p}\right)= & \frac{(t+1)^{p}+(t+2)^{p}+\cdots+(t+t)^{p}}{t^{p+1}} \\
\leq & \frac{\frac{(2 t)^{p+1}}{p+1}+\frac{(2 t)^{p}}{2}+O(2 t)^{p-1}}{t^{p+1}} \\
& =\frac{2^{p+1}}{p+1}+\frac{2^{p}}{2 t}+O\left(\frac{2^{p}}{t^{2}}\right)
\end{aligned}
$$


The $2^{p+1} / p+1$ term is exactly the lower bound we used in Lemma 9 , and we now have an idea of how much error this introduced:

$$
\begin{aligned}
E\left(\left(\frac{t_{k+1}}{t_{k}}\right)^{n-k-1}\right) & =\frac{2^{n-k}}{n-k}+\frac{2^{n-k}}{4 t_{k}}+O\left(\frac{2^{n-k}}{t_{k}^{2}}\right) \\
& =E\left(u_{k}^{n-k-1}\right)\left(1+\frac{n-k}{4 t_{k}}+O\left(\frac{n-k}{t_{k}^{2}}\right)\right)
\end{aligned}
$$

The expected value now depends on $t_{k}$, and to bound the error, we need some idea of how large we expect $t_{k}$ to be.

Consider again the continuous analogue used in the proof of Lemma 9. We expect $s_{k}$ to grow exponentially, as $c^{k}$ for some $c$, so look at the distribution of $s_{k}^{1 / k}$. Taking logs, we see that $\log s_{k}^{1 / k}$ is distributed as the average of $k$ copies of $\log x$ on $(1,2]$, each with mean $2 \log 2-1$. Thus as $k$ increases without bound, the distribution of $s_{k}^{1 / k}$ converges towards a point distribution at $4 / e$. As we already noted, the $t_{k}$ certainly grow no slower than the $s_{k}$. We conclude that for any constant $c<4 / e$ and probability $p<1$, there is a sufficiently large $k_{0}$ such that $\operatorname{Pr}\left(t_{k}>c^{k}\right)>p$ for all $k \geq k_{0}$.

The error we want to bound is the product of the $n$ error terms $e_{k}=1+\frac{n-k}{4 t_{k}}+O\left(\frac{n-k}{t_{k}^{2}}\right)$ for $k=1, \ldots, n$. To simplify bookkeeping, we will instead think about $\lg s(n)$ and bound the sum of the logs of the error terms. We separate the work into two cases, depending on whether $k$ is less or greater than $2 \log n$.

When $k<2 \log n$, the denominator $t_{k}$ may be small, and the error terms $\log e_{k}$ may be as much as $O(\log n)$. Adding up all $2 \log n$ of these terms gives a total which is $O(\log n)^{2}$, the error term in the statement of our theorem.

Finally, when $k=2 \log n$, we know that as long as $n$ is sufficiently large, with high probability $t_{k}>c^{2 \log n}$, which is on the order of $n^{2}$. Thus with high probability, $e_{k}$ is $1+O(1 / n)$, and $\log e_{k}=O(1 / n)$. Since the error terms monotonically decrease, the sum of all the terms with $k \geq 2 \log n$ is bounded by $n e_{2} \log n=O(1)$. So taking $n$ sufficiently large ensures that the error in the lower bound is concentrated almost entirely in the first $2 \log n$ error terms, and we are done.

Computational evidence based on the actual values of $s(n)$ for $n$ up to 190 indicates that the constant needed to make $\lg s(n)<\left(\begin{array}{l}n \\ 2\end{array}\right)-\lg n !+c(\log n)^{2}$ reaches a peak of $c \approx 1.18304060 \ldots$ at $n=32$ and decreases slowly thereafter.

\section{References}

[1] Bach, E.; Shallit, J. Algorithmic Number Theory, vol. 1. MIT Press, Cambridge, MA, 1996.

[2] Banderier, C.; Bousquet-Mélou, M.; Denise, A.; Flajolet, P.; Gardy, D.; GouyouBeauchamps, D. Generating functions for generating trees. Preprint, 2000. 
[3] Barcucci, E.; Del Lungo, A.; Pergola, E; Pinzani, R. ECO: a methodology for the enumeration of combinatorial objects. J. Differ. Equations Appl. 5 (1999), no. 4-5, 435-490.

[4] Capelli, P.; Narayana, T. V. On Knock-Out Tournaments. Canad. Math. Bull. 13 (1970), 105-109

[5] Chung, F. R. K.; Graham, R. L.; Hoggatt, V. E., Jr.; Kleiman, M. The number of Baxter permutations. J. Combin. Theory Ser. A 24 (1978), 382-394.

[6] Fishburn, P. C.; Odlyzko, A. M. Unique subjective probability of finite sets. J. Ramanujan Math. Soc. 4 (1989), 1-23

[7] Finch, S. Conjectures about 1-additive sequences. Fibonacci Quart. 29 (1991), 209214.

[8] Knuth, D. Estimating the Efficiency of Backtrack Programs. Math. Comput. 29 \#129 (1975), 121-136.

[9] Sloane, N. J. A. Sloane's On-Line Encyclopedia of Integer Sequences. http://www.research.att.com/ njas/sequences/

[10] Torelli, M. Increasing Integer Sequences and Goldbach's Conjecture. Preprint, 1996.

[11] Tromp, J.; Shallit, J. Subword complexity of a generalized Thue-Morse word. Information Processing Lett. 54 (1995), 313-316.

[12] West, J. Generating trees and the Catalan and Schröder numbers. Discrete Math. 146 (1995), 247-262.

[13] West, J. Generating trees and forbidden subsequences. Proceedings of the 6th Conference on Formal Power Series and Algebraic Combinatorics (New Brunswick, NJ, 1994). Discrete Math. 157 (1996), 363-374. 